\title{
Surveillance of Ruminant Diseases in the Nordic Countries
}

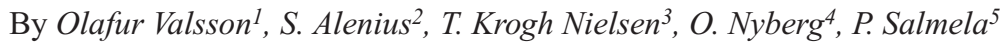 \\ ${ }^{1}$ Dalvik Distrikt Veterinarian,Eyrarlandvegur 24, 600 Akureyri, Iceland, ${ }^{2}$ National Veterinary Institute, P.O.Box \\ 7073, SE-75007 Uppsala, Sweden, ${ }^{3}$ Danish Veterinary Laboratory, Bülowsvej 27, DK-1790 Copenhagen V, \\ Denmark, ${ }^{4}$ Veterinærinstituttet, P.O. Box 8156 dep, NO-0033 Oslo, Norway, ${ }^{5}$ Lihakunta Pl 147, SF-70101 Kuo- \\ pio, Finland.
}

\begin{abstract}
All the Nordic countries have a basis for their surveillance and disease control in ruminants in national legislation and regulations listing notifiable diseases of concern to the countries. The Nordic countries are a disease- free zone comparing to other parts of the world and the aim of the surveillance is to keep that status and be able to document it. Following is a short summary from each country.
\end{abstract}

In Denmark, exotic diseases that have never been diagnosed or at least detected decades ago are surveyed to state freedom. At the present time the cattle population is surveyed systematically for three diseases: IBR (free since 1992), enzootic leucosis (free since 1991), and BVD (eradication program since 1994). Surveillance for TSE (BSE and scrapie) is based on diseased animals with central nervous signs. In sheep and goats Maedi-Visna and CAE are controlled through a voluntary program started in 1979. Sheep and goats are monitored for Brucella melitensis.

In Finland the cattle population is surveyed to state that the country is free from disease for; IBR, enzootic bovine leucosis, BSE, Brucellosis and Tuberculosis, and surveillance for disease control is performed for BVD, and Salmonellosis. Sheep and goats are surveyed for Scrapie, Maedi-Visna, CAE and Brucellosis.

None of the known bovine viral diseases have ever been diagnosed in Iceland. Samples for IBR, BVD and enzootic bovine leucosis have been taken on a sporadic ground from cattle due to export, all with negative result. Surveillance on Paratuberculosis is through samples taken from every adult cow and sheep slaughtered and samples taken on suspicion. Scrapie status is surveyed through samples from every adult sheep slaughtered, life inspection in Scrapie-areas and veterinary inspection on suspicion. Maedi-visna was eradicated by stamping out from the icelandic sheep population.

Norway has, on a historical background, Brucellosis-free status. A surveillance system to confirm this will be set up in 1999. Surveillance to document a disease free status is performed on IBR, enzootic bovine leucosis and BSE. Surveillance for control and status of disease is performed on paratuberculosis, enterohemorrhagic escerichia coli, salmonellaand BVD. The ultimate goal for BVD surveillance is a disease free status. A very extensive surveillance system is operated on scrapie and Maedi-Visna in small ruminants.

The Swedish cattle population is at present considered free from IBR, BSE, brucellosis and bovine tuberculosis. The aim of the surveil- 
lance for these diseases is to monitor, protect and keep this disease free status. A national eradication programme for BLV was introduced in Sweden in 1990. The aim of the programme is to reach a disease free status. A national voluntary programme for the control of BVD was introduced in 1993. The final aim of this programme is that Sweden should reach a disease free status concerning BVD.Salmonella infections are surveyed to keep the present low prevalence of these infections. During 1998 a voluntary programme against bovine paratuber- culosis in beef cattle started with the aim of preventing the spread of the disease and if possible reach a disease free status for the entire cattle population. A voluntary control programme for Maedi-Visna in sheep started in 1993 to control the disease A similar programme for CAE in goats is planned to start during 1999. The Swedish sheep population is considered free from scrapie and brucellosis and the aim of the surveillance is to monitor and keep this disease free status. 